\title{
Errata zum Artikel von Axel Wiebrecht in Chinesische Medizin 1/2015
}

Bedauerlicherweise sind in zwei Tabellen des Artikels von Axel Wiebrecht „Mögliche Gentoxizität chinesischer Arzneidrogen und Konsequenzen für die Schwangerenbehandlung " in der Ausgabe Chinesische Medizin Nr.1/2015, S. 21-37, einige Fehler unterlaufen. Wir bringen hier die korrigierten Tabellen 2 und 4.

Tabelle 2: Experimentelle Ergebnisse zu Gentoxizität, Mutagenität und Cancerogenität Schwangerschafts-relevanter Drogen. Ergebnisse aus dem Ames-Test, aus sonstigen Tests an Bakterien, an Säugetierzellen, aus Tierversuchen bzw. aus Cancerogenitätstests in Langzeit-Tierversuchen. Ein ${ }^{\prime-}{ }^{\prime \prime}$ zeigt ein negatives, ein ", ${ }^{\prime \prime}$ ein positives Testergebnis an. Werte in Klammern sind nur schwach positiv oder haben verminderte Aussagekraft, z.B. weil nur fraktionierte Bestandteile der Drogen getestet oder die Testsubstanzen parenteral verabreicht wurden. Eine weitere Wertung der Testergebnisse hinsichtlich ihrer Validität ist nicht aufgenommen. Für den Ames-Test wurde jeder getestete Bakterienstamm einzeln gezählt, ohne Unterscheidung, ob mit oder ohne metabolische Aktivierung. Wenn der Test mit oder ohne metabolische Aktivierung positiv war, wurde der Bakterienstamm als positiv gekennzeichnet.,?" bei Lycii fr. bedeutet, dass nicht klar ist, ob fructus oder cortex gemeint ist.

\begin{tabular}{|c|c|c|c|c|c|c|}
\hline Droge & Ames & Bakterien & Säugetierz. & Tierversuch & Cancerog. & Quelle \\
\hline \multicolumn{7}{|l|}{ Albiziae Cort. } \\
\hline \multicolumn{7}{|l|}{ Albiziae Fl. } \\
\hline \multicolumn{7}{|l|}{ Amomi Fr. } \\
\hline Angelicae sinens. Rd. & ----- & & - & ---- & & {$[12-15]$} \\
\hline Asini corii Colla & ----- & & & -- & & {$[15,16]$} \\
\hline Astragali Rd. & --------(-)+ & & -- & --------+++ & & {$[12,13,17-22]$} \\
\hline Atractylodis macroc. Rhz. & ----- & & -- & --- & & {$[12,23]$} \\
\hline Bupleuri Rd. & $--(-)$ & & - & -- & & {$[12,18]$} \\
\hline Chuanxiong Rhz. & ------ & & - & --- & & {$[12,24]$} \\
\hline Citri reticulatae Peric. & -- & & & -- & & {$[12]$} \\
\hline Codonopsis Rd. & --- & & & -- & & {$[12,15]$} \\
\hline Coicis Sem. & $---(-)$ & - & - & -- & & {$[12,25,26]$} \\
\hline Corni Fr. & --- & - & & ----- & & {$[26-28]$} \\
\hline Cuscutae Sem. & --+ & & - & $--(+)(+)+$ & & {$[12,25,29,30]$} \\
\hline \multicolumn{7}{|l|}{ Dipsaci Rd. } \\
\hline \multicolumn{7}{|l|}{ Ecliptae Hb. } \\
\hline Eucommiae Cort. & ---------- & & --+ & $--------(+)(+)$ & & {$[12,14,15,31-36]$} \\
\hline Glehniae Rd. & -- & - & & & & {$[26]$} \\
\hline \multirow[t]{2}{*}{ Glycyrrhizae Rd. } & ---------- & --+ & --- & ----- & & {$[12,15,17,26,37-42]$} \\
\hline & $--(-)(-)$ & & & & & \\
\hline Lycii Fr. & $(-)(--?)$ & - & - & -- & & {$[12,25,39]$} \\
\hline Ophiopogonis Rd. & ---- & - & & -- & & {$[12,26]$} \\
\hline Paeoniae Rd. alba & ------- & $(+)$ & & --- & - & {$[12,17,43,44]$} \\
\hline Pinelliae Rhz. praep. & $---(-)$ & & - & & & {$[30,45]$} \\
\hline Poria & -- & - & & --- & & {$[26,46-48]$} \\
\hline Rehmanniae Rd. & $--(-)$ & & -- & -- & & {$[12,18,49]$} \\
\hline Rehmanniae Rd. praep. & --- & & & $(+)(+)$ & & {$[12,15]$} \\
\hline Schisandrae Fr. & --------(-) & -+ & - & -- & & {$[15,25,26,50]$} \\
\hline Taxilli Herb. & & & & $(+)(+)$ & & {$[51]$} \\
\hline \multirow[t]{2}{*}{ Zingiberis Rhz. (recens) } & ----------(+) & $---(+)+$ & - & $----(+)(+)$ & $--(+)$ & {$[17,26,52-60]$} \\
\hline & $(+)++++$ & & & & & \\
\hline Ziziphi spinosae Sem. & ---- & & & - & & {$[61]$} \\
\hline
\end{tabular}


Tabelle 4: Ergebnisse verschiedener Testverfahren zur Gentoxizität bzw. Mutagenität von Zingiberis Rhizoma (recens). Ergebnisse im AmesTest, aus Tests an sonstigen Bakterien, an Säugetierzellen, aus Tierversuchen und aus Cancerogenitätsstudien in Langzeit-Tierversuchen. Ein ,-" zeigt ein negatives, ein ${ }_{\|}+$" ein positives Testergebnis an. Mut $\downarrow$ bedeutet eine antimutagene Wirkung gegenüber einem bekannten Mutagen, Mut \pm eine ausbleibende antimutagene Wirkung und Mut $\uparrow$ eine Verstärkung der Wirkung eines bekannten Mutagens. Werte in Klammern sind nur schwach positiv, ein ${ }_{\text {}}+!$ " bedeutet stark positiv. Für den Ames-Test wurde jeder getestete Bakterienstamm einzeln aufgeführt, der jeweils erste Wert ohne, der zweite mit


ethanolisch. MNT=micronucleus test, $C O M=$ comet assay, CAT=chromosome aberration test, Blasen $(\uparrow)=$ gesteigerte Blasen-Carcinogenität zweier Cancerogene in einer Untergruppe, Mamma-/Intest.-=keine erhöhte Rate von spontanen Mamma- bzw. intestinalen Tumoren, Haut $\downarrow=$ inhibitorischer Effekt auf die Promotion von Hauttumoren bei der Maus unter topischer Verabreichung.

\begin{tabular}{|c|c|c|c|c|c|c|}
\hline Substrat/Extrakt & Ames & Bakterien & Säugetierz. & in vivo & Cancerog. & Quelle \\
\hline frisch, w/m & $--/--$ & & & & & [17] \\
\hline getrocknet, w/m & $--/++$ & & & & & [17] \\
\hline getrocknet, w & & & & & Mamma- & [59] \\
\hline frisch, w & & & & CAT+ & & {$[54]$} \\
\hline Paste/Pulver, w & --/-- & & & & & {$[56]$} \\
\hline Pulver, w & & & & Mut $\pm / \downarrow$ & & {$[56]$} \\
\hline$w-a$ & & & COM- & MNT- & $\operatorname{Blasen}(\uparrow)$ & {$[55]$} \\
\hline Saft, w-löslich & & Mut $\downarrow$ & & & & {$[52]$} \\
\hline Saft & & $-/$ Mut $\uparrow$ & & & & {$[52]$} \\
\hline Saft, ä & & Mut \pm & & & & {$[52]$} \\
\hline Pulver & & & & COM-,MNT- & & {$[58]$} \\
\hline 70\%-a & $(+)(+)$ & Mut $\downarrow \downarrow$ & & & & {$[57]$} \\
\hline frisch, a & & & & & Haut $\downarrow$ & {$[60]$} \\
\hline frisch, ä-a & $--/--/-+/++$ & & & & & {$[53]$} \\
\hline Gingerol & --/--/-+/-+ & Mut $\uparrow /+!$ & $\begin{array}{l}\text { COM+, } \\
\text { MNT+ }\end{array}$ & & $\begin{array}{l}\text { Haut } \downarrow \text {, } \\
\text { Intest.- }\end{array}$ & {$[44,52,53,65-67]$} \\
\hline Zingeron & --/--/--/-- & & & & & {$[53]$} \\
\hline
\end{tabular}

